\title{
STOCHASTICITY IN MODELS OF ELLIPTICAL GALAXIES
}

\author{
Daniel Pfenniger, Stéphane Udry \\ Geneva Observatory \\ CH-1290 Sauverny \\ Switzerland
}

\begin{abstract}
We measure quantitatively the growth of stochasticity due to various perturbations to a model of elliptical galaxy. This is achieved by computing the Liapunov characteristic exponents of randomly selected orbits.
\end{abstract}

\section{INTRODUCTION}

The amount of stochasticity has important implications on the effective orbital relaxation time, which is much shorter for stochastic orbits (Pfenniger, 1986) than classically thought. We examine here the nature of stellar motions by computing orbits in a relatively realistic triaxial model. Stochasticity is measured by computing the Liapunov characteristic exponents (hereafter LCE) of many randomly selected orbits. The effects of changing the axis ratios, rotation speeds or adding various perturbations to the potential can then be estimated quantitatively. The principal differences of our investigation with an analogous work of Gerhard (1985) are first that the orbits here are arbitrary and move in $3 \mathrm{D}$, and secondly that the potentials we have retained are not chosen to be nearly integrable, but to be as much as possible in accordance with the profile of elliptical galaxies.

\section{MODELS AND PERTURBATIONS}

The retained triaxial density law is $\rho(x, y, z)=\rho_{c} /\left(1+\frac{x^{2}}{a^{2}}+\frac{y^{2}}{b^{2}}+\frac{z^{2}}{c^{2}}\right)^{n}, n=\frac{3}{2}$, which is close to Schwarzschild's model (1979) but is exactly ellipsoidal. The potential $\Phi$ of this density stratification, called hereafter $P_{3 / 2}$, takes a simple form in the confocal ellipsoidal coordinates $\lambda, \mu, \nu$ (de Zeeuw, 1984):

$$
\Phi(x, y, z)=-2 \pi G \rho_{c} a b c \int_{0}^{\infty} \frac{d u}{\sqrt{(u+\lambda)(u+\mu)(u+\nu)}} .
$$

Contrarily to the perfect ellipsoid of de Zeeuw, for which $n=2$, this potential is nonintegrable. It is presently a good compromise between the actual density profile of elliptical galaxies and the need to compute orbits with a not too expensive scheme. This is achieved by using the algorithms $R_{F}$ and $R_{D}$ of Carlson (1979). 
We have considered the following models up to now:

$M_{1.0}$ : The $P_{3 / 2}$ potential with the Schwarzschild axis ratios $(a / b / c)=(8 / 5 / 4)$ and a rotation corresponding to a corotation radius sets at 32 .

$M_{1.1}$ : As $M_{1.0}$ but with an additional central Plummer potential of radius $r_{p}=0.01$, and a fractional mass of $2 \%$.

$M_{1.2}$ : As $M_{1.0}$ but with an additional $\Phi_{3}=\epsilon \cos 3 \theta$ azimuthal perturbation of amplitude $\epsilon=0.0008$,

$M_{1.3}$ : As $M_{1.0}$ but with an additional $\Phi_{4}=\epsilon \cos 4 \theta$ perturbation.

$M_{2 . i}$ : As $M_{1 . i}, i=0 . .3$ but with the axis ratios of $(8 / 4 / 2.5)$.

The LCE $\chi_{i}$ of a given orbit express the average rate of divergence of the other neighbouring orbits, over a relevant time. The $\chi_{i}$ also express the sensitivity to perturbations. In $3 \mathrm{D}$ potentials, there are 6 of them which occur in 3 pairs $\left(\chi_{i},-\chi_{i}\right)$. To every isolating integral corresponds a pair of vanishing LCE, therefore regular orbits are especially robust to perturbations. The Kolmogorov entropy $h$, i.e. the rate at which the information about the initial conditions of an orbit is lost, is just equal to the sum of the positive exponents: $h=\sum_{\chi_{i}>0} \chi_{i}$ (Pesin, 1977). This quantity is used below for measuring stochasticity. Its inverse is the typical time-scale for losing the information about the initial conditions.

\section{COMPUTATION OF STOCHASTICITY}

We have computed the LCE of 100 orbits per model over a time slightly larger than the Hubble time $t_{H}$, starting with the same set of random initial conditions. A global estimation of stochasticity is given by the average $h$ of the 100 orbits, $<h>$. Another way to estimate the amount of stochasticity is to count the fraction of orbits with a $h$ larger than some value. $\Gamma_{0.0015}$ is the fraction of orbits for which $h>0.0015$. Since $t_{\langle h\rangle}=\langle h\rangle^{-1}$ is a time-scale, it can be compared to the Hubble time $t_{H}$. The computations are summarized in the following table.

\begin{tabular}{|c|cccc|cccc|}
\hline Models & $M_{1.0}$ & $M_{1.1}$ & $M_{1.2}$ & $M_{1.3}$ & $M_{2.0}$ & $M_{2.1}$ & $M_{2.2}$ & $M_{2.3}$ \\
\hline$<h>\times 10^{3}$ & 1.19 & 2.21 & 2.53 & 3.52 & 1.23 & 2.91 & 2.86 & 3.55 \\
$\Gamma_{0.0015}$ & 0.06 & 0.70 & 0.80 & 1.00 & 0.07 & 0.76 & 0.90 & 1.00 \\
$t_{<h>} / t_{H}$ & 0.28 & 0.15 & 0.13 & 0.09 & 0.27 & 0.11 & 0.11 & 0.09 \\
\hline
\end{tabular}

For all the orbits considered, the perturbations systematically increase $h$ with respect to the corresponding orbits in the unperturbed models $M_{1.0}$ or $M_{2.0}$. The average time-scale for the loss of information $t_{\langle h\rangle}$ is always shorter than the Hubble time, especially for the perturbed models.

\section{REFERENCES}

Carlson, B.C.: 1979, Numer. Math. 33, 1

Gerhard, O.E.: 1985, Astron. Astrophys. 151, 279

Pesin, Ya.B.: 1977, Russ. Math. Surveys 32, 55

Pfenniger, D.: 1986, Astron. Astrophys. 165, 74

Schwarzschild, M.: 1979, Astrophys. J. 232, 236

de Zeeuw, P.T.: 1984, Thesis, Leiden University 